Bull. Korean Math. Soc. 51 (2014), No. 6, pp. 1805-1827

http://dx.doi.org/10.4134/BKMS.2014.51.6.1805

\title{
CERTAIN CLASSES OF ANALYTIC FUNCTIONS AND DISTRIBUTIONS WITH GENERAL EXPONENTIAL GROWTH
}

\author{
Byung Keun Sohn
}

\begin{abstract}
Let $\mathcal{K}_{M}^{\prime}$ be the generalized tempered distributions of $e^{M(t)}$ growth, where the function $M(t)$ grows faster than any linear functions as $|t| \rightarrow \infty$, and let $\mathbf{K}_{\mathbf{M}}^{\prime}$ be the Fourier transform spaces of $\mathcal{K}_{M}^{\prime}$. We obtain the relationship between certain classes of analytic functions in tubes, $\mathcal{K}_{M}^{\prime}$ and $\mathbf{K}_{\mathbf{M}}^{\prime}$.
\end{abstract}

\section{Introduction}

In his book [9], V. S. Vladimirov has considered the relationship between the class of analytic functions in tubes $H(A ; C)$ and tempered distributions with polynomial growth $\mathcal{S}^{\prime}$. Later R. D. Carmichael [3] has introduced two different types of classes of analytic functions in tubes $G_{p}(A ; C)$ and $F_{p}(A ; C)$ both of which are extensions of $H(A ; C)$ and has obtained the relationship between $G_{p}(A ; C)$ (and $F_{p}(A ; C)$ ) and tempered distributions with exponential growth of polynomial powers $\mathcal{K}_{p}^{\prime}, p>1$, and the Fourier transform spaces $\mathbf{K}_{\mathbf{p}}^{\prime}, p>1$ of $\mathcal{K}_{p}^{\prime}, p>1$.

In this paper, we introduce two different types of classes of analytic functions in tubes $G_{M}(A ; C)$ and $F_{M}(A ; C)$ which are extensions of $G_{p}(A ; C)$ and $F_{p}(A ; C)$, respectively, and obtain the relationship between $G_{M}(A ; C)$ (and $\left.F_{M}(A ; C)\right)$ and tempered distributions with general exponential powers growth $\mathcal{K}_{M}^{\prime}$ and the Fourier transform $\mathbf{K}_{\mathrm{M}}^{\prime}$ of $\mathcal{K}_{M}^{\prime}$.

In the main sections, we show that elements of $G_{M}(A ; C)$ and $F_{M}(A ; C)$ can be represented as the Fourier-Laplace transform of distributions $\mathcal{K}_{M}^{\prime}$. Also we present representations of $G_{M}(A ; C)$ and $F_{M}(A ; C)$ as elements in $\mathbf{K}_{\mathbf{M}}^{\prime}$ in terms of Fourier transforms in $\mathbf{K}_{\mathrm{M}}^{\prime}$ of certain elements in $\mathcal{K}_{M}^{\prime}$ and strong boundedness for $G_{M}(A ; C)$ and $F_{M}(A ; C)$ as elements in $\mathbf{K}_{\mathbf{M}}^{\prime}$. In particular, we show that elements of $F_{M}(A ; C)$ obtain distributional boundary values in $\mathbf{K}_{\mathbf{M}}^{\prime}$.

Received November 18, 2013; Revised July 9, 2014.

2010 Mathematics Subject Classification. 46F20, 46F12, 46F10.

Key words and phrases. analytic functions, distributions. 


\section{Notation and preliminaries}

We denote the points of $\mathbb{R}^{n}$ spaces by $t=\left(t_{1}, t_{2}, \ldots, t_{n}\right)$ and $s=\left(s_{1}, s_{2}, \ldots\right.$, $\left.y_{n}\right)$. The letter $n$ always denotes the dimension. In $\mathbb{C}^{n}$ the points are denoted by $z=x+i y, x, y \in \mathbb{R}^{n}$. We define $\langle t, s\rangle=t_{1} s_{1}+t_{2} s_{2}+\cdots+t_{n} s_{n}$ and similarly define $\langle t, z\rangle, t \in \mathbb{R}^{n}, z \in \mathbb{C}^{n}$. $\alpha$ denotes $n$ tuples $\left(\alpha_{1}, \alpha_{2}, \ldots, \alpha_{n}\right)$ of nonnegative integers. $|\alpha|=\alpha_{1}+\alpha_{2}+\cdots+\alpha_{n}$ and $\alpha !=\alpha_{1} ! \alpha_{2} ! \cdots \alpha_{n}$ !. If $k=\left(k_{1}, k_{2}, \ldots, k_{n}\right)$ is an $n$ tuples of integers, $t^{k}=t_{1}^{k_{1}} t_{2}^{k_{2}} \cdots t_{n}^{k_{n}}, t \in \mathbb{R}^{n}$, with similar definition for $z^{k}, z \in \mathbb{C}^{n}$. If $a \in R$, then $a t=\left(a t_{1}, a t_{2}, \ldots, a t_{n}\right)$. We write $D_{j}=-\frac{1}{2 \pi i}\left(\frac{\partial}{\partial t_{j}}\right), j=1,2, \ldots, n$, and $D_{t}^{\alpha}=D_{1}^{\alpha_{1}} D_{2}^{\alpha_{2}} \cdots D_{n}^{\alpha_{n}}$ and similarly write $D_{z}^{\alpha}$.

Definition 1. A set $C \subset \mathbb{R}^{n}$ is a cone with vertex at zero if $y \in C$ implies $\lambda y \in C$ for all positive real scalar $\lambda$.

Definition 2. Let $C$ be a cone. $C \cap\left\{y \in \mathbb{R}^{n}:|y|=1\right\}$ is called the projection of $C$ and is denoted $\operatorname{pr}(C)$.

Definition 3. If $C^{\prime}$ and $C$ are cones such that $\operatorname{pr}\left(\bar{C}^{\prime}\right) \subset \operatorname{pr}(C)$, then $C^{\prime}$ is called a compact subcone of $C$.

For a cone $C, \mathcal{O}(c)$ will denote the convex hull or envelop of $C$ and $T^{C}=$ $\mathbb{R}^{n}+i C \subseteq \mathbb{C}^{n}$ is a tube in $\mathbb{C}^{n}$

Definition 4. If $C$ is open, $T^{C}$ is called a tubular cone. If $C$ is both open and connected, $T^{C}$ is called a tubular radial domain.

Definition 5. The set $C^{*}=\left\{t \in \mathbb{R}^{n}:\langle t, y\rangle \geq 0, y \in C\right\}$ is the dual cone of the cone $C$ and $C_{*}=\mathbb{R}^{n} \backslash C^{*}$.

Definition 6. The function

$$
u_{C}=\sup _{y \in \operatorname{pr}(C)}(-\langle t, y\rangle)
$$

is the indicatrix of the cone $C$.

It follows that $C^{*}=\left\{t \in \mathbb{R}^{n}: u_{C}(t) \leq 0\right\}$. Further $u_{C}(t) \leq u_{\mathcal{O}(C)}(t)$ and if $t \in C^{*}$, then $u_{C}(t)=u_{\mathcal{O}(C)}(t)[9$, p. 219]. To characterize the nonconvexity of the cone, we have the following; for a cone $C$, let

$$
\rho_{C}=\sup _{t \in C^{*}} \frac{u_{\mathcal{O}(C)}}{u_{C}(t)} .
$$

A cone $C$ is convex if and only if $\rho_{C}=1$ [9, Sec. 25.1, Lemma 2] and if a cone is open and consists of finite number of components, then $\rho_{C}<1[9$, Sec. 25.1, Lemma 3]. In this paper we shall be considering the case $1 \leq \rho_{C}<+\infty$ for all cones $C$.

Now we present four important facts what will be used frequently later.

Lemma 1 ([9, Sec. 25.1]). Let $C$ be a cone. Then

$$
-\langle t, y\rangle \leq|y| u_{\mathcal{O}(C)}, \quad u_{\mathcal{O}(C)} \leq \rho_{C} u_{C}(t), \quad t \in C^{*}, y \in \mathcal{O}(C) .
$$


Lemma 2 ([9, Eq.(28), p. 241]). Let $C$ be an open connected cone and let $C_{*}^{\prime}$ be a compact subcone of $C_{*}$. Then there exist $\xi=\xi\left(C_{*}^{\prime}\right)$, depending on $C_{*}^{\prime}$, such that

$$
\xi|t| \leq u_{C}(t) \leq|t|, \quad t \in C_{*}^{\prime} .
$$

Lemma 3 ([9, Sec. 25.2, Lemma 2]). Let $C$ be an open cone and $C^{\prime}$ that is an arbitrary subcone of $\mathcal{O}(C)$. Then there exist a number $\delta=\delta\left(C^{\prime}\right)$ and open cone $\left(C^{*}\right)^{\prime}$ both depending on $C^{\prime}$ such that $C^{*} \subset\left(C^{*}\right)^{\prime}$ and

$$
\langle y, t\rangle \geq \delta|y||t|, \quad y \in C^{\prime} \subset \mathcal{O}(C), t \in\left(C^{*}\right)^{\prime} .
$$

Lemma 4 ([9, Lemma, p. 241]). Let $C_{*}^{\prime}$ be cone that is compact in the cone $C_{*}$. For an arbitrary number $\eta \in(0,1)$, there exists a compact subcone $C^{\prime}=$ $C^{\prime}\left(C_{*}^{\prime}, \eta\right)$ depending on $C_{*}^{\prime}$ and on $\eta$ such that for any $t \in C_{*}^{\prime}$, there exists a point $y_{t}^{0} \in \operatorname{Pr}\left(C^{\prime}\right)$ at which

$$
-\left\langle t, y_{t}^{0}\right\rangle \geq(1-\eta) u_{C}(t)
$$

\section{The distribution spaces $\mathcal{K}_{M}^{\prime}$ and $\mathrm{K}_{\mathrm{M}}^{\prime}$}

Let $\mu(\xi), 0 \leq \xi \leq \infty$, denote a continuous increasing function such that $\mu(0)=0, \mu(\infty)=\infty$. For $t \geq 0$ we define

$$
M(t)=\int_{0}^{t} \mu(\xi) d \xi .
$$

The function $M(t)$ is an increasing, convex, and continuous function with $M(0)=0$ and $M(\infty)=\infty$. Further we define $M(t)$ for negative $t$ by $M(-t)=$ $M(t)$. Since the derivative $\mu(t)$ of $M(t)$ is unbounded in $R$, the function $M(t)$ will grow faster than any linear function as $|t| \rightarrow \infty$.

The function $M(t)$ can be defined on $\mathbb{R}^{n}$ by $M\left(t_{1}+t_{2}+\cdots+t_{n}\right)=M\left(t_{1}\right)+$ $M\left(t_{2}\right)+\cdots+M\left(t_{n}\right)$ for all $t=\left(t_{1}, t_{2}, \ldots, t_{n}\right) \in \mathbb{R}^{n}$. (Refer to Sec. 4.1 of Chapter 1 in [4] or p. 130 in [5].)

Definition 7. Let $M(x)$ and $\Omega(y)$ be the functions corresponding to $\mu(\xi)$ and $\omega(\eta)$ as above, respectively. Then $M(x)$ and $\Omega(y)$ are called to be dual in the sense of Young if $\mu(\omega(\eta))=\eta$ and $\omega(\mu(\xi))=\xi$.

We have two examples of dual functions in the sense of Young as follow;

$$
\begin{aligned}
& \text { 1. } M(s)=\frac{s^{p}}{p}, \quad \Omega(t)=\frac{t^{q}}{q}, \frac{1}{p}+\frac{1}{q}=1, \quad s, t \geq 0 . \\
& \text { 2. } M(s)=e^{s}-s-1, \Omega(t)=(t+1) \log (t+1)-t, \quad s, t \geq 0 .
\end{aligned}
$$

We list some properties of function $M(x), x \in \mathbb{R}^{n}$.

Lemma 5. For $t \geq 0$ we define $M(t)=\int_{0}^{t} \mu(\xi) d \xi$, where $\mu(\xi)(0 \leq \xi \leq \infty)$ is a continuous increasing function such that $\mu(0)=0$ and $\mu(\infty)=\infty$. Then we have that

$$
M(s)+M(t) \leq M(s+t) \text { for all st } \geq 0
$$




$$
M\left(\frac{t}{k}\right) \leq \frac{M(t)}{k} \text { for all } k>1
$$

Hence if we let $x=\left(x_{1}, x_{2}, \ldots, x_{n}\right)$ and $y=\left(y_{1}, y_{2}, \ldots, y_{n}\right)$ be in $\mathbb{R}^{n}$, then

$$
\begin{aligned}
& M(x)+M(y) \leq M(x+y) \text { for all } x_{i} y_{i} \geq 0,(i=1,2, \ldots, n) . \\
& M\left(\frac{x}{k}\right) \leq \frac{M(x)}{k} \text { for all } k>1 \text { and } x \in \mathbb{R}^{n} .
\end{aligned}
$$

We note an important property of dual functions which will be useful later.

Lemma 6 ([5, Lemma 1.1]). Let $M(s)$ and $\Omega(t)$ be defined as in Definition 7, where $s, t \in R$. Then

$$
s t \leq M(s)+\Omega(t) \text { for any } s, t \geq 0
$$

and the equality holds if and only if $t=\mu(s)$ or $s=\omega(t)$.

Hence if we let $x=\left(x_{1}, x_{2}, \ldots, x_{n}\right)$ and $y=\left(y_{1}, y_{2}, \ldots, y_{n}\right)$ be in $\mathbb{R}^{n}$, then

$$
\langle x, y\rangle \leq M(x)+\Omega(y) \text { for any } x_{i}, y_{i} \geq 0,(i=1,2, \ldots, n)
$$

and the equality holds if and only if $y_{i}=\mu\left(x_{i}\right)$ or $x_{i}=\omega\left(y_{i}\right)$.

For more details about the function $M(x)$ and $\Omega(y)$, we can refer to $[4$, Chapter 1].

Using the function $M(t)$, we define the space $\mathcal{K}_{M}$ as the space of all functions $\varphi(t)$ in $C^{\infty}$ such that

$$
\nu_{k}(\varphi)=\sup _{t \in \mathbb{R}^{n},|\alpha| \leq k} e^{M(k t)}\left|D_{t}^{\alpha} \varphi(t)\right|<\infty, k=1,2, \ldots,
$$

where $D_{t}^{\alpha}=D_{1}^{\alpha_{1}} D_{2}^{\alpha_{2}} \cdots D_{n}^{\alpha_{n}}$ and $D_{j}^{\alpha_{j}}=-\frac{1}{2 \pi i} \frac{\partial^{\alpha_{j}}}{\left(\partial t_{j}\right)^{\alpha_{j}}}$ and $|\alpha|=\alpha_{1}+\alpha_{2}+$ $\cdots+\alpha_{n}$. The topology in $\mathcal{K}_{M}$ is defined by the countably family of semi-norms $\left\{\nu_{k}\right\}_{k=1}^{\infty}$. It follows that the space $\mathcal{K}_{M}$ becomes a Fréchet space [5] and the identity mapping $\mathcal{D} \hookrightarrow \mathcal{K}_{M} \hookrightarrow \mathcal{E}$ are continuous when $\mathcal{E}$ denotes the space of all $C^{\infty}$ functions on $\mathbb{R}^{n}$ and $\mathcal{D}$ the space of all $C^{\infty}$ functions with compact support in $\mathbb{R}^{n}$.

Lemma 7. $\mathcal{K}_{M}$ is a Montel space.

Proof. If $B$ is a bounded set of $\mathcal{K}_{M}$, then $B$ is a bounded set in $C^{\infty}$ since the imbedding $\mathcal{K}_{M} \hookrightarrow C^{\infty}$ is continuous. Since $C^{\infty}$ is a Montel space, it suffices to show that $B$ is a relatively compact set in $C^{\infty}$. Let $\left(\phi_{j}\right)$ be a sequence of elements of $B$ such that $\left(\phi_{j}\right)$ converges to $\phi$ in $C^{\infty}$. Since $B$ is a bounded set of $\mathcal{K}_{M}$, for all $k \in N$ and all $\alpha \in N^{n}$, there exists a constant $C_{k, \alpha}$ such that

$$
\sup _{t \in \mathbb{R}^{n}}\left|e^{M(k t)} D^{\alpha} \phi_{j}(t)\right| \leq C_{k, \alpha}, \quad \phi_{j} \in B .
$$

The inequality (1) implies that, given $\epsilon>0$ there is a constant $M>0$ such that for $t$ with $|t|>M$,

$$
\left|e^{M(k t)} D^{\alpha} \phi_{j}(t)\right| \leq \epsilon, \quad \phi_{j} \in B .
$$


Since $\phi_{j} \rightarrow \phi$ in $C^{\infty},(2)$ implies that

$$
\left|e^{M(k t)} D^{\alpha} \phi(t)\right| \leq \epsilon, \quad|t|>M .
$$

Hence $\phi \in \mathcal{K}_{M}$. On the other hand, since $\phi_{j} \rightarrow \phi$ in $C^{\infty},\left(D^{\alpha} \phi_{j}\right)$ converges uniformly to $D^{\alpha} \phi$ on the compact set $\left\{t \in \mathbb{R}^{n}:|t| \leq M\right\}$. This implies that given $\epsilon>0$, we can find an integer $j_{0}$ such that

$$
e^{M(k t)}\left|D^{\alpha} \phi_{j}(t)-D^{\alpha} \phi(t)\right| \leq \epsilon
$$

for all $t$ with $|t| \leq M$ and all $j \geq j_{0}$. Last three inequalities imply that

$$
\sup _{t \in \mathbb{R}^{n}} e^{M(k t)}\left|D^{\alpha} \phi_{j}(t)-D^{\alpha} \phi(t)\right| \leq \epsilon
$$

for all $j \geq j_{0}$; therefore $\phi_{j} \rightarrow \phi$ in $\mathcal{K}_{M}$. The proof is completed.

We denote by $\mathcal{K}_{M}^{\prime}$ the space of all continuous linear functional on $\mathcal{K}_{M}$. Clearly when $M(t)=\log (1+|t|), \mathcal{K}_{M}^{\prime}$ is the space of Schwartz's tempered distributions. When $M(t)=|t|, \mathcal{K}_{M}^{\prime}$ is the space of tempered distributions of $\mathcal{K}_{1}^{\prime}$ which is introduced and characterized by J. Sevastião E. Silva [8]. When $M(t)=|t|^{p}, p>1, \mathcal{K}_{M}^{\prime}$ is the space of tempered distributions of $\mathcal{K}_{p}^{\prime}, p>1$, which is introduced and characterized by Sampson and Zielezny [6].

The restriction $\tilde{T}=\left.T\right|_{\mathcal{D}}$ of a functional $T \in \mathcal{K}_{M}^{\prime}$ to $\mathcal{D}$ is a distribution. Since $\mathcal{D}$ is dense in $\mathcal{K}_{M}, T$ is determined by its values on $\mathcal{D}$. We characterize the distributions in $\mathcal{K}_{M}^{\prime}$ by their growth at infinity.

Lemma 8 ([5, Theorem 2.3]). A distribution $T \in \mathcal{D}$ is in $\mathcal{K}_{M}^{\prime}$ if and only if there exist positive integers $k, \alpha$ and a bounded continuous function $f(t)$ on $\mathbb{R}^{n}$ such that

$$
T=D^{\alpha}\left[e^{M(k t)} f(t)\right]
$$

Let $\phi(t) \in L^{1}\left(\mathbb{R}^{n}\right)$. We define the Fourier transform of $\phi(t)$ by

$$
\hat{\phi}(x)=\mathcal{F}[\phi(t) ; x]=\int_{\mathbb{R}^{n}} \phi(t) e^{2 \pi i\langle x, t\rangle} d t
$$

and the inverse Fourier transform of $\phi(t)$ by

$$
\mathcal{F}^{-1}[\phi(t) ; x]=\int_{\mathbb{R}^{n}} \phi(t) e^{-2 \pi i\langle x, t\rangle} d t .
$$

Now we have a Paley-Wiener type theorem for the space $\mathcal{K}_{M}$ from [5, Theorem 4.1]; an entire function $F(\zeta)$ is a Fourier transform of a function $\varphi$ in $\mathcal{K}_{M}$ if and only if, for every integer $N \geq 0$ and every $\epsilon>0$, there exists a constant $C$ such that

$$
|F(\xi+i \eta)| \leq C(1+|\zeta|)^{-N} e^{\Omega(\epsilon \eta)}, \quad \zeta=\xi+i \eta \in \mathbb{C}^{n} .
$$

Let $\mathbf{K}_{\mathbf{M}}$ be the space of Fourier transform of functions in $\mathcal{K}_{M}$. We define in $\mathbf{K}_{\mathbf{M}}$ a locally convex topology by means of the seminorms

$$
\omega_{k}(\hat{\varphi})=\sup _{\zeta=\xi+i \eta}(1+|\zeta|)^{k} e^{-\Omega\left(\frac{\eta}{k}\right)} \mid \hat{\varphi}(\zeta), k=1,2, \ldots, \varphi \in \mathcal{K}_{M}
$$


Lemma 9 ([5, Coro. 4.2]). The Fourier transform is a topological isomorphism of $\mathcal{K}_{M}$ onto $\mathbf{K}_{\mathbf{M}}$.

Let $\mathbf{K}_{\mathbf{M}}^{\prime}$ be the space of continuous linear functional on $\mathcal{K}_{M}$ which equipped with the topology of uniform convergence on all bounded set in $\mathbf{K}_{\mathbf{M}}$. Each distribution $T$ in $\mathcal{K}_{M}^{\prime}$ has a Fourier transform $\hat{T}$ in $\mathbf{K}_{\mathbf{M}}^{\prime}$ defined by Parseval's formula

$$
\langle\hat{T}, \hat{\varphi}\rangle=(2 \pi)^{n}\langle T, \varphi\rangle, \quad \varphi \in \mathcal{K}_{M} .
$$

Moreover, we have:

Lemma 10 ([5, Coro. 4.3]). The Fourier transform is a topological isomorphism of $\mathcal{K}_{M}^{\prime}$ onto $\mathbf{K}_{\mathbf{M}}^{\prime}$.

For further detailed structure theories about $\mathcal{K}_{M}^{\prime}$ and $\mathbf{K}_{\mathbf{M}}^{\prime}$, we can refer to [4] and [5].

\section{The analytic spaces $G_{M}(A ; C)$ and $F_{M}(A ; C)$}

To find the relations between the increase in certain classes of analytic functions and the properties of their spectral functions, Vladimirov [9, Sec. 26.4] introduced the following class of analytic functions;

Let $C$ be an open cone in $\mathbb{R}^{n}$ and $C^{\prime}$ be an arbitrary compact subcone of $C$. $p \geq 1$ and $A \geq 0$ are real numbers. A function $f(z)$ belongs to the class $\mathbf{H}_{\mathbf{p}}(\mathbf{A} ; \mathbf{C})$ if $f(z)$ is analytic in the tubular cone $T^{C}=\mathbb{R}^{n}+i C \subset \mathbb{C}^{n}$ and satisfies

$$
|f(z)| \leq K\left(C^{\prime}\right)(1+|z|)^{N}(1+|y|)^{-M} e^{A|y|^{p}}, \quad z=x+i y \in T^{C},
$$

where $K\left(C^{\prime}\right)$ is a constant depending on $C^{\prime}$, and $N$ and $M$ are nonnegative real numbers which do not depend on $C^{\prime}$.

Motivated by the works of Vladimirov, R. D. Carmichael introduced two different types of classes of analytic functions in tubes both of which are more general spaces than the class $H_{p}(A ; C)$ as follow;

Let $C$ be an open cone in $\mathbb{R}^{n}$ and $C^{\prime}$ be an arbitrary compact subcone of $C$. $p \geq 1$ and $A \geq 0$ are real numbers. Let $m>0$. $T\left(C^{\prime} ; m\right)$ denotes the set $T\left(C^{\prime} ; m\right)=\mathbb{R}^{n}+i\left(C^{\prime} \backslash\left(C^{\prime} \cap N(0, m)\right)\right)$ where $N(0, m)$ is a closed ball in $\mathbb{R}^{n}$ of radius $m>0$ with center at the origin. A function $f(z)$ belongs to the class $\mathbf{G}_{\mathbf{p}}(\mathbf{A} ; \mathbf{C})$ if, for each compact subcone $C^{\prime} \subset C$, there exists a fixed $m=m\left(C^{\prime}\right)>0$ depending on $C^{\prime}$ such that $f(z)$ is analytic in $T\left(C^{\prime} ; m\right)$ and satisfies

$$
|f(z)| \leq K\left(C^{\prime} ; m\right)(1+|z|)^{N} e^{2 \pi A|y|^{p}}, \quad z=x+i y \in T\left(C^{\prime} ; m\right),
$$

where $K\left(C^{\prime} ; m\right)$ is a constant depending on $C^{\prime}$ and on $m$ and $N$ is a nonnegative real number which does not depend on $C^{\prime}$ and on $m$.

A function $f(z)$ belongs to $\mathbf{F}_{\mathbf{p}}(\mathbf{A} ; \mathbf{C})$ if, for each compact subcone $C^{\prime} \subset C$, $f(z)$ is analytic in $T^{C^{\prime}}=\mathbb{R}^{n}+i C^{\prime}$ and satisfies

$$
|f(z)| \leq K\left(C^{\prime} ; m\right)(1+|z|)^{N} e^{2 \pi A|y|^{p}}, \quad z=x+i y \in T\left(C^{\prime} ; m\right),
$$


where $K\left(C^{\prime} ; m\right)$ is a constant depending on $C^{\prime}$ and on $m$ and $N$ is a nonnegative real number which does not depend on $C^{\prime}$ and on $m$.

Carmichael studied the relationship between $G_{p}(A ; C)$ (and $F_{p}(A ; C)$ ), the distributions $\mathcal{K}_{p}^{\prime}, p \geq 1$, and the Fourier transform $\mathbf{K}_{p}^{\prime}, p \geq 1$, of $\mathcal{K}_{p}^{\prime}, p \geq 1$, in [3]. Since $\mathcal{K}_{p}^{\prime} \subset \mathcal{K}_{M}^{\prime}, p \geq 1$, we need more general classes of analytic functions than $G_{p}(A: C)$ or $F_{p}(A ; C)$ to find the relationship between the classes of analytic functions, $\mathcal{K}_{M}^{\prime}$, and $\mathbf{K}_{\mathrm{M}}^{\prime}$ as follow;

For $t \geq 0$, let $M(t)=\int_{0}^{t} \mu(\xi) d \xi$, where $\mu(\xi)(0 \leq \xi \leq \infty)$ is a continuous increasing function such that $\mu(0)=0$ and $\mu(\infty)=\infty$. Let $C$ be an open cone in $\mathbb{R}^{n}$ and $C^{\prime}$ be an arbitrary compact subcone of $C . A \geq 0$ are real numbers. Let $m>0 . T\left(C^{\prime} ; m\right)$ denote the set $T\left(C^{\prime} ; m\right)=\mathbb{R}^{n}+i\left(C^{\prime} \backslash\left(C^{\prime} \cap N(0, m)\right)\right)$ where $N(0, m)$ is a closed ball in $\mathbb{R}^{n}$ of radius $m>0$ with center at the origin.

A function $f(z)$ belongs to the class $\mathbf{G}_{\mathbf{M}}(\mathbf{A} ; \mathbf{C})$ if, for each compact subcone $C^{\prime} \subset C$, there exists a fixed $m=m\left(C^{\prime}\right)>0$ depending on $C^{\prime}$ such that $f(z)$ is analytic in $T\left(C^{\prime} ; m\right)$ and satisfies

$$
|f(z)| \leq K\left(C^{\prime} ; m\right)(1+|z|)^{N} e^{2 \pi M(A y)}, \quad z=x+i y \in T\left(C^{\prime} ; m\right),
$$

where $K\left(C^{\prime} ; m\right)$ is a constant depending on $C^{\prime}$ and on $m$ and $N$ is a nonnegative real number which does not depend on $C^{\prime}$ and on $m$.

A function $f(z)$ belongs to $\mathbf{F}_{\mathbf{M}}(\mathbf{A} ; \mathbf{C})$ if, for each compact subcone $C^{\prime} \subset C$, $f(z)$ is analytic in $T^{C^{\prime}}=\mathbb{R}^{n}+i C^{\prime}$ and satisfies

$$
|f(z)| \leq K\left(C^{\prime} ; m\right)(1+|z|)^{N} e^{2 \pi M(A y)}, \quad z=x+i y \in T\left(C^{\prime} ; m\right),
$$

where $K\left(C^{\prime} ; m\right)$ is a constant depending on $C^{\prime}$ and on $m$ and $N$ is a nonnegative real number which does not depend on $C^{\prime}$ and on $m$.

The $2 \pi$ in the exponential term in the definition of $G_{M}(A ; C)$ and $F_{M}(A ; C)$ simply reflects the way we have defined the Fourier transform in this paper. Obviously we have the following inclusion relation;

$$
F_{M}(A ; C) \subset G_{M}(A ; C), \quad G_{p}(A ; C) \subset G_{M}(A ; C), \quad F_{p}(A ; C) \subset F_{M}(A ; C) .
$$

We need three lemmas which will be useful to obtain main results in the next two sections.

Lemma 11. For $t \geq 0$, let $\Omega(t)=\int_{0}^{t} \omega(\xi) d \xi$, where $\omega(\xi)(0 \leq \xi \leq \infty)$ is a continuous increasing function such that $\omega(0)=0$ and $\omega(\infty)=\infty$. Let $C$ be an open connected cone and let $C_{*}^{\prime}$ be an arbitrary compact subcone of $C_{*}=\mathbb{R}^{n} \backslash C^{*}$. Let $\gamma$ be an $n$-tuple of nonnegative integers. Let $n \geq 1$ be an integer and let $R>0$. Then we have

$$
(1+|t|)^{n+1+|\gamma|} \leq M_{1} \exp \left[2 \pi R \Omega\left(u_{C}(t)\right)\right],
$$

where $M_{1}=M_{1}\left(C_{*}^{\prime}\right)$ depends on $C_{*}^{\prime} \subset C_{*}$.

Hence for $A>0$

$$
(1+|t|)^{n+1+|\gamma|} \leq M_{2} \exp \left[2 \pi R \Omega\left(\frac{u_{C}(t)}{A}\right)\right],
$$


where $M_{2}=M_{2}\left(C_{*}^{\prime}, A\right)$ depends on $C_{*}^{\prime} \subset C_{*}$ and on $A$.

Proof. From Lemma 2, given $C_{*}^{\prime} \subset C_{*}$ there exists $\xi=\xi\left(C_{*}^{\prime}\right)$, depending on $C_{*}^{\prime}$, such that

$$
\xi|t| \leq u_{C}(t) \leq|t|, \quad t \in C_{*}^{\prime} .
$$

Hence, for any $R>0$,

$$
0<\exp [2 \pi R \Omega(\xi t)] \leq \exp \left[2 \pi R \Omega\left(u_{C}(t)\right)\right], \quad t \in C_{*}^{\prime} .
$$

Since the function $\Omega(t)$ in the hypothesis grows faster than any linear function as $|t| \rightarrow \infty$ for $t \in \mathbb{R}^{n}$,

$$
(1+|t|)^{-n-1-|\gamma|} \exp [2 \pi R \Omega(\xi t)] \rightarrow \infty \text { as }|t| \rightarrow \infty
$$

for $t \in \mathbb{R}^{n}$, hence

$$
(1+|t|)^{-n-1-|\gamma|} \exp \left[2 \pi R \Omega\left(u_{C}(t)\right)\right] \rightarrow \infty \text { as }|t| \rightarrow \infty
$$

for $t \in C_{*}^{\prime} \subset C_{*}$. Let $N(0, m)$ be a closed ball of the origin in $\mathbb{R}^{n}$ of radius $m>0$. We can find $O_{m}>1$, depending on $m$, such that

$$
Q_{m}(1+|t|)^{-n-1-|\gamma|} \exp [2 \pi R \Omega(\xi t)] \geq 1, \quad t \in N(0, m) .
$$

By Lemma 2 and (7),

$$
Q_{m}(1+|t|)^{-n-1-|\gamma|} \exp \left[2 \pi R \Omega\left(u_{C}(t)\right)\right] \geq 1, \quad t \in N(0, m) \cap C_{*}^{\prime} .
$$

Thus we have (3) from (6) and (8). Now if $A>0$, we have from (5) that given $C_{*}^{\prime} \subset C_{*}$, there exists $\xi=\xi\left(C_{*}^{\prime}\right)$, depending on $C_{*}^{\prime}$, such that

$$
\frac{\xi|t|}{A} \leq \frac{u_{C}(t)}{A} \leq \frac{|t|}{A}, \quad t \in C_{*}^{\prime} .
$$

If we replace (5) by (9), we have from the same process as above that

$$
\left(1+\left|\frac{t}{A}\right|\right)^{n+1+|\gamma|} \leq M_{1} \exp \left[2 \pi R \Omega\left(\frac{u_{C}(t)}{A}\right)\right]
$$

But since $(1+|t|) \leq C_{1}(1+|t| / A)$ when $C_{1}=C_{1}(A)$, depending on $A$, equals $A$ if $A \geq 1$ and equals 1 if $0<A<1$, we have (4) from (10).

Lemma 12. For $t \geq 0$, let $\Omega(t)=\int_{0}^{t} \omega(\xi) d \xi$, where $\omega(\xi)(0 \leq \xi \leq \infty)$ is a continuous increasing function such that $\omega(0)=0$ and $\omega(\infty)=\infty$. Let $C$ be an open connected cone and let $C^{\prime}$ be an arbitrary open compact subcone of $\mathcal{O}(C)$. Let $C_{*}^{\prime}$ be an arbitrary compact subcone of $C_{*}=\mathbb{R}^{n} \backslash C^{*}$. Let $A>0$. Let $g(t)$ be a continuous function of $t \in \mathbb{R}^{n}$ which satisfies

$$
|g(t)| \leq K\left(C_{*}^{\prime}, \eta\right) \exp \left[-2 \pi(1-2 \eta) \Omega\left(\frac{u_{C}(t)}{A}\right)\right], t \in C_{*}^{\prime} \subset C_{*},
$$

for any $\eta \in(0,1)$ with $1-3 \eta>0$, where $K\left(C_{*}^{\prime}, \eta\right)$ is a constant, depending on $C_{*}^{\prime}$ and on $\eta$. Let $z_{0} \in T^{C^{\prime}}=\mathbb{R}^{n}+i C^{\prime}$ be an arbitrary but fixed point and let 
$z \in N^{\prime}\left(z_{0}, r\right) \subset T^{C^{\prime}}$, where $N^{\prime}\left(z_{0}, r\right)$ is an open neighborhood of $z_{0}$ with radius $r>0$ whose closure is in $T^{C^{\prime}}$. Then for any $n$-tuple $\gamma$ of nonnegative integer,

$$
h_{C_{*}^{\gamma}}^{\gamma, g}(z)=\int_{C_{*}^{\prime}} t^{\gamma} g(t) e^{2 \pi i\langle z, t\rangle} d t
$$

converges absolutely and uniformly for $z \in N^{\prime}\left(z_{0}, r\right)$.

Proof. From Lemma 1 and assumption about the estimation of $g(t)$, for $z=$ $x+i y \in N^{\prime}\left(z_{0}, r\right)$, there exists a real number $T$ with $|y|=|\operatorname{Im}(z)| \leq T$ such that for $A>0$ and any $\eta>0$ with $1-2 \eta>0$

$$
\begin{aligned}
&\left|h_{C_{*}^{\prime}}^{\gamma, g}(t)\right| \leq K\left(C_{*}^{\prime}, \eta\right) \int_{C_{*}^{\prime}}\left|t^{\gamma}\right| e^{-2 \pi\langle y, t\rangle} \\
& \cdot \exp \left[-2 \pi(1-2 \eta) \Omega\left(\frac{u_{C}(t)}{A}\right)\right] d t \\
& \leq K\left(C_{*}^{\prime}, \eta\right) \int_{C_{*}^{\prime}} \frac{(1+|t|)^{n+1+|\gamma|}}{(1+|t|)^{n+1}} \exp \left[2 \pi T \rho_{C} u_{C}(t)\right] \\
& \cdot \exp \left[-2 \pi(1-2 \eta) \Omega\left(\frac{u_{C}(t)}{A}\right)\right] d t
\end{aligned}
$$

where $n$ is the dimension.

By Lemma 2 and Lemma 11 with $R=\eta$, for $t \in C_{*}^{\prime} \subset C_{*}$, there exists a $\xi=\xi\left(C_{*}^{\prime}\right)$ such that

$$
\begin{aligned}
& \exp \left[2 \pi T \rho_{C} u_{C}(t)-2 \pi(1-2 \eta) \Omega\left(\frac{u_{C}(t)}{A}\right)\right] \cdot(1+|t|)^{n+1+|\gamma|} \\
\leq & M\left(C_{*}^{\prime}, A\right) \exp \left[2 \pi T \rho_{C} u_{C}(t)-2 \pi(1-2 \eta) \Omega\left(\frac{u_{C}(t)}{A}\right)\right] \\
& \cdot \exp \left[2 \pi \eta \Omega\left(\frac{u_{C}(t)}{A}\right)\right] \\
\leq & M\left(C_{*}^{\prime}, A\right) \exp \left[2 \pi T \rho_{C}|t|-2 \pi(1-3 \eta) \Omega\left(\frac{\xi t}{A}\right)\right] .
\end{aligned}
$$

Now consider the function of $x$ defined by

$$
f(x)=2 \pi T \rho_{C} x-2 \pi(1-3 \eta) \Omega\left(\frac{\xi x}{A}\right), x>0 .
$$

Since $\Omega(x)=\int_{0}^{x} \omega(t) d t$, we have $\Omega^{\prime}(x)=\omega(x)$, hence

$$
f^{\prime}(x)=2 \pi T \rho_{C}-2 \pi(1-3 \eta) \omega\left(\frac{\xi x}{A}\right) .
$$

Since $\omega(x)$ is a continuous increasing function, $\omega(x)$ has its inverse function $\omega^{-1}(x)$. Hence if we take $\eta \in(0,1), f(x)$ attains its maximum at

$$
x=\frac{A}{\xi} \cdot \omega^{-1}\left(\frac{T \rho_{C}}{1-3 \eta}\right)>0 .
$$


Hence, for $t \in C_{*}^{\prime} \subset C_{*}$, if we take $\eta \in(0,1)$ with $1-3 \eta>0$,

$$
\begin{aligned}
& \exp \left[2 \pi T \rho_{C}|t|-2 \pi(1-3 \eta) \Omega\left(\frac{\xi t}{A}\right)\right] \\
\leq & \exp \left[2 \pi T \rho_{C} \frac{A}{\xi} \cdot \omega^{-1}\left(\frac{T \rho_{C}}{1-3 \eta}\right)-2 \pi(1-3 \eta) \Omega\left(\omega^{-1}\left(\frac{T \rho_{C}}{1-3 \eta}\right)\right)\right] .
\end{aligned}
$$

Thus we have from (11), (12), and (13) that

$$
\begin{aligned}
\left|h_{C_{*}^{\prime}}^{\gamma, g}(z)\right| \leq & M\left(C_{*}^{\prime}, A\right) K\left(C_{*}^{\prime}, \eta\right) \exp \left[2 \pi T \rho_{C} \frac{A}{\xi} \cdot \omega^{-1}\left(\frac{T \rho_{C}}{1-3 \eta}\right)\right. \\
& \left.-2 \pi(1-3 \eta) \Omega\left(\omega^{-1}\left(\frac{T \rho_{C}}{1-3 \eta}\right)\right)\right] \cdot \int_{C_{*}^{\prime}} \frac{1}{(1+|t|)^{n+1}} d t \\
\leq & K^{\prime}\left(C_{*}^{\prime}, A, \eta\right) \exp \left[2 \pi T \rho_{C} \frac{A}{\xi} \cdot \omega^{-1}\left(\frac{T \rho_{C}}{1-3 \eta}\right)\right. \\
& \left.-2 \pi(1-3 \eta) \Omega\left(\omega^{-1}\left(\frac{T \rho_{C}}{1-3 \eta}\right)\right)\right]
\end{aligned}
$$

for all $z \in N^{\prime}\left(z_{0}, r\right)$, where $K^{\prime}\left(C_{*}^{\prime}, A, \eta\right)$ is a constant depending on fixed $C_{*}^{\prime}$, on fixed $A>0$, and on fixed $\eta \in(0,1)$ with $1-3 \eta>0$. Since the last term of (14) is independent of $z \in N^{\prime}\left(z_{0}, r\right)$, the function $h_{C^{\prime}}^{\gamma, g}(z)$ converges absolutely and uniformly for $z \in N^{\prime}\left(z_{0}, r\right)$.

Remark. The estimation of inequalities in (14) will be continued under some additional conditions in Theorem 2 of the next section.

Lemma 13. Let $C$ be an open connected cone and let $C^{\prime}$ be an arbitrary open compact subcone of $\mathcal{O}(C)$. Let $\left(C^{*}\right)^{\prime}$ be an open cone as in Lemma 3 and let $C_{*}^{\prime}=\mathbb{R}^{n} \backslash\left(C^{*}\right)^{\prime} \subset C_{*}$. Let $z_{0} \in T\left(C^{\prime} ; m\right)=\mathbb{R}^{n}+i\left(C^{\prime} \backslash\left(C^{\prime} \cap N(0, m)\right)\right)$ be arbitrary but fixed and let $z \in N^{\prime}\left(z_{0}, r\right) \subset T\left(C^{\prime} ; m\right)$, where $N^{\prime}\left(z_{0}, r\right)$ is an open neighborhood of $z_{0}$ with radius $r>0$ whose closure is in $T\left(C^{\prime} ; m\right)$. Let $g(t)$ satisfies

$$
|g(t)| \leq K e^{k|t|}, t \in\left(C^{*}\right)^{\prime}
$$

for some constants $K$ and $k \geq 0$. Then for any $n$-tuple $\gamma$ of nonnegative integers,

$$
h_{\left(C_{*}\right)^{\prime}}^{\gamma, g}(z)=\int_{\left(C_{*}\right)^{\prime}} t^{\gamma} g(t) e^{2 \pi i\langle z, t\rangle} d t
$$

converges absolutely and uniformly for $z \in N^{\prime}\left(z_{0}, r\right)$.

Proof. By Lemma 3, there exist a number $\delta=\delta\left(C^{\prime}\right)$ and an open cone $\left(C^{*}\right)^{\prime}$ both depending on $C^{\prime}$ such that $C^{*} \subset\left(C^{*}\right)^{\prime}$ and

$$
\langle y, t\rangle \geq \delta|y||t|, \quad y \in C^{\prime} \subset \mathcal{O}(C), t \in\left(C^{*}\right)^{\prime} .
$$

We choose the real number $m=m\left(C^{\prime}\right)>0$ depending on $C^{\prime}$ such that

$$
m=\frac{k}{(2 \pi \delta)}+1
$$


where $k \geq 0$ is as in (15). Then if $y \in C^{\prime}$ with $|y|>m, k-2 \pi \delta|y|<-2 \pi \delta<0$. For the chosen $m>0$ in (17), let $z_{0}$ be an arbitrary but fixed point in $T\left(C^{\prime} ; m\right)$. Choose $N^{\prime}\left(z_{0}, r\right)$ whose closure is in $T\left(C^{\prime} ; m\right)$. Then we have from (16) and (17) that for $z \in N^{\prime}\left(z_{0}, r\right)$,

$$
\begin{aligned}
& \left|h_{C_{*}^{\prime}}^{\gamma, g}(z)\right| \\
= & \left|\int_{\left(C^{*}\right)^{\prime}} t^{\gamma} g(t) e^{2 \pi i\langle z, t\rangle} d t\right| \\
\leq & K \int_{\left(C^{*}\right)^{\prime}}\left|t^{\gamma}\right| e^{k|t|} e^{-2 \pi\langle y, t\rangle} d t \\
\leq & K \int_{\left(C^{*}\right)^{\prime}}\left|t^{\gamma}\right| \exp [(k-2 \pi \delta|y|)|t|] d t \leq K \int_{\left(C^{*}\right)^{\prime}}\left|t^{\gamma}\right| \exp [-2 \pi \delta|t|] d t \\
\leq & K Z_{n} \int_{0}^{\infty} s^{|\gamma|+n-1} \exp [-2 \pi \delta s] d s=K Z_{n}(|\gamma|+n-1) !(2 \pi \delta)^{-|\gamma|-n} .
\end{aligned}
$$

Here we have used [7, Theorem 32, p. 39] in the second to last step in (18) and integration by parts $(|\gamma|+n-1)$ times in the last step in (18), where $K$ is the constant as in (15) and $Z_{n}$ is the area of the unit sphere in $\mathbb{R}^{n}$. Since the last term of (18) is independent of $z \in N^{\prime}\left(z_{0}, r\right)$, the function $h_{C_{*}^{\prime}}^{\gamma, g}(z)$ converges absolutely and uniformly for $z \in N^{\prime}\left(z_{0}, r\right)$.

\section{The relationship $G_{M}(A ; C), \mathcal{K}_{M}^{\prime}$, and $\mathrm{K}_{\mathrm{M}}^{\prime}$}

In this section, we show that elements of $G_{M}(A ; C)$ can be represented as the Fourier-Laplace transform of distributions $\mathcal{K}_{M}^{\prime}$. Also we present representations of $G_{M}(A ; C)$ as elements in $\mathbf{K}_{\mathbf{M}}^{\prime}$ in terms of Fourier transforms in $\mathbf{K}_{\mathbf{M}}^{\prime}$ of certain elements in $\mathcal{K}_{M}^{\prime}$ and strong boundedness for $G_{M}(A ; C)$ as elements in $\mathbf{K}_{\mathbf{M}}^{\prime}$.

Theorem 1. Let $M(x)$ and $\Omega(y)$ be the functions as in Definition 7 . For the open connected cone $C$, let $f(z) \in G_{M}(A ; C)$. For any compact subcone $C^{\prime} \subset C$, let $m=m\left(C^{\prime}\right)$ be a fixed real number which depends on $C^{\prime}$ as in the definition of $G_{M}(A ; C)$. Then there exist a unique element $V=D_{t}^{\alpha}(g(t)) \in$ $\mathcal{K}_{M}^{\prime}$, where $\alpha$ is an n-tuple of nonnegative integers and $g(t)$ is a continuous function of $t \in \mathbb{R}^{n}$ such that the following are hold.

(I) For $A \geq 0$

$$
f(z)=z^{\alpha} \mathcal{F}\left[e^{-2 \pi\langle y, t\rangle} g(t) ; x\right], \quad z=x+i y \in T\left(C^{\prime} ; m\right),
$$

where the Fourier transform is taken in the $L^{2}$ sense.

(II) For $A \geq 0, g(t)$ satisfies

$$
|g(t)| \leq K\left(C^{\prime}, m\right) \exp [2 \pi(M(A y)+|y||t|)], \quad t \in \mathbb{R}^{n},
$$

where $C^{\prime} \subset C$ is arbitrary and $K\left(C^{\prime}, m\right)$ depends on $C^{\prime}$ and on $m$. Inequality (20) is independent of $y \in\left(C^{\prime} \backslash\left(C^{\prime} \cap N(0, m)\right)\right)$ and $\operatorname{supp}(g)=\operatorname{supp}(V) \subseteq\{t$ : $\left.u_{C}(t) \leq A\right\}$. 
(III) For $A>0$ and any compact subcone of $C_{*}^{\prime} \subset C_{*}=\mathbb{R}^{n} \backslash C^{*}, g(t)$ satisfies

$$
|g(t)| \leq M\left(C_{*}^{\prime}, \eta\right) \exp \left[-2 \pi(1-2 \eta) \Omega\left(\frac{u_{C}(t)}{A}\right)\right], t \in C_{*}^{\prime},
$$

where any $\eta \in(0,1)$ is such that $1-2 \eta>0$ and $M\left(C_{*}^{\prime}, \eta\right)$ is a constant depending on $C_{*}^{\prime}$ and on $\eta$.

(IV) Let $A \geq 0$. If $g(t)$ satisfies that $|g(t)| \leq K e^{k|t|}$ for any $t \in\left(C^{*}\right)^{\prime}$ and for some constants $K$ and $k>0$, then

$$
f(z)=\left\langle V, e^{2 \pi i\langle z, t\rangle}\right\rangle, \quad z=x+i y \in T\left(C^{\prime} ; m\right) .
$$

(V) For $A \geq 0$,

$$
f(z)=\mathcal{F}\left[e^{-2 \pi\langle y, t\rangle} V_{t}\right], \quad z=x+i y \in T\left(C^{\prime} ; m\right),
$$

where the equality in (23) holds in $\mathbf{K}_{\mathbf{M}}^{\prime}$.

(VI)

$$
\left\{f(z): y=\operatorname{Im}(z) \in\left(C^{\prime} \backslash\left(C^{\prime} \cap N(0, m)\right)\right),|y| \leq Q_{m}\right\}
$$

is strongly bounded in $\mathbf{K}_{\mathbf{M}}^{\prime}$, where $Q_{m}>m>0$.

Proof. Let $C$ be an open connected cone and let $C^{\prime}$ be an arbitrary open compact subcone of $C$. For any compact subcone $C^{\prime} \subset C$, let $m=m\left(C^{\prime}\right)$ be a fixed real number which depends on $C^{\prime}$ as in the definition of $G_{M}(A ; C)$ corresponding to $f(z)$. Since $f(z) \in G_{M}(A ; C)$, we can choose an $n$-tuple $\alpha$ of nonnegative integers which is independent of $C^{\prime}$ and of $m$ such that for $z=x+i y \in T\left(C^{\prime} ; m\right)$ and $\epsilon>0$,

$$
\left|z^{-\alpha} f(z)\right| \leq K^{\prime}\left(C^{\prime} ; m\right)(1+|z|)^{-n-\epsilon} e^{2 \pi M(A y)},
$$

where $K^{\prime}\left(C^{\prime} ; m\right)$ is a constant and $n$ is a dimension. Put

$$
g(t)=\int_{\mathbb{R}^{n}} z^{-\alpha} f(z) e^{-2 \pi i\langle z, t\rangle} d x, \quad z=x+i y \in T\left(C^{\prime} ; m\right),
$$

which is a continuous function of $t \in \mathbb{R}^{n}$. By [2, Theorem 1, p. 846] and (25), $g(t)$ is independent of $y=\operatorname{Im}(z) \in\left(C^{\prime} \backslash\left(C^{\prime} \cap N(0, m)\right)\right)$.

Proof of (I). We have from (25) that $z^{-\alpha} f(z) \in L_{1} \cap L_{2}$ as a function of $x=\operatorname{Re}(z)$ for an arbitrary $y \in\left(C^{\prime} \backslash\left(C^{\prime} \cap N(0, m)\right)\right)$. Thus from (26),

$$
e^{-2 \pi\langle y, t\rangle} g(t)=\mathcal{F}^{-1}\left[z^{-\alpha} f(z) ; t\right], \quad z=x+i y \in T\left(C^{\prime} ; m\right),
$$

where the Fourier transform is taken in the $L_{2}$ sense. By the Plancherel theorem, $e^{-2 \pi\langle y, t\rangle} g(t) \in L_{2}$ and

$$
z^{-\alpha} f(z)=\mathcal{F}\left[e^{-2 \pi\langle y, t\rangle} g(t) ; x\right], \quad z=x+i y \in T\left(C^{\prime} ; m\right),
$$

where the Fourier transform is taken in the $L_{1}$ or $L_{2}$ sense. This complete the proof of (I).

Proof of (II). From (25) and (26),

$$
|g(t)| \leq K^{\prime}\left(C^{\prime} ; m\right) e^{2 \pi M(A y)} e^{2 \pi\langle y, t\rangle} \int_{\mathbb{R}^{n}}(1+|x|)^{-n-\epsilon} d x
$$




$$
\leq K^{\prime \prime}\left(C^{\prime} ; m\right) \exp [2 \pi(M(A y)+\langle y, t\rangle)],
$$

where $K^{\prime \prime}\left(C^{\prime} ; m\right)$ is a constant. Since $g(t)$ is independent of $y=\operatorname{Im}(z) \in$ $\left(C^{\prime} \backslash\left(C^{\prime} \cap N(0, m)\right)\right),(29)$ holds independently of $y=\operatorname{Im}(z) \in\left(C^{\prime} \backslash\left(C^{\prime} \cap\right.\right.$ $N(0, m))$ ). From exactly the same process in [1, pp. 846-847], we have that $\operatorname{supp}(g)=\operatorname{supp}(V) \subseteq\left\{t: u_{C}(t) \leq A\right\}$. This complete the proof of (II).

Consider

$$
V=D_{t}^{\alpha}(g(t)) .
$$

Since $g(t)$ is a continuous function and satisfies (II), $g(t) \in \mathcal{K}_{M}^{\prime}$ by Lemma 8 , hence $V=D_{t}^{\alpha}(g(t)) \in \mathcal{K}_{M}^{\prime}$. In fact $V=D_{t}^{\alpha}(g(t)) \in \mathcal{K}_{1}^{\prime} \subset \mathcal{K}_{p}^{\prime}, p>1$.

Proof of (III). Let $C_{*}^{\prime}$ be an arbitrary but fixed compact subcone of $C_{*}$. By Lemma 4 , for any $\eta \in(0,1)$, there exists a compact subcone $C^{\prime}=C^{\prime}\left(C_{*}^{\prime}, \eta\right)$ of $C \subset \mathcal{O}(C)$, depending on $C_{*}^{\prime}$ and on $\eta$, such that we can find a point $y_{t}^{0} \in \operatorname{Pr}\left(C^{\prime}\right)$ where

$$
-\left\langle t, y_{t}^{0}\right\rangle \geq(1-\eta) u_{C}(t)
$$

for any $t \in C_{*}^{\prime}$. Put

$$
y_{t}=\frac{1}{A} y_{t}^{0}\left|M^{-1}\left(\Omega\left(\frac{u_{C}(t)}{A}\right)\right)\right| .
$$

Since $C^{\prime}$ is a cone and $y_{t}^{0} \in \operatorname{Pr}\left(C^{\prime}\right), y_{t} \in C^{\prime} \subset C$ for any $t \in C_{*}^{\prime}$. Choose a real number $R>0$ such that

$$
R>\frac{A\left(\Omega^{-1}(M(A m))\right)}{\xi},
$$

where $m=m\left(C^{\prime}\right)$ is as in the definition of $G_{M}(A ; C)$ corresponding to $f(z)$ and $\xi=\xi\left(C_{*}^{\prime}\right)$ is as in Lemma 2. Then for $t \in C_{*}^{\prime}$ with $|t|>R>0$, we have from Lemma 2, (31), and (32) that

$$
\begin{aligned}
\left|y_{t}\right| & =\frac{1}{A}\left|M^{-1}\left(\Omega\left(\frac{u_{C}(t)}{A}\right)\right)\right| \geq \frac{1}{A}\left|M^{-1}\left(\Omega\left(\frac{\xi t}{A}\right)\right)\right| \\
& \geq \frac{1}{A}\left|M^{-1}\left(\Omega\left(\frac{\xi R}{A}\right)\right)\right| \geq m .
\end{aligned}
$$

Hence if $t \in C_{*}^{\prime}$ with $|t|>R>0, y_{t} \in\left(C^{\prime} \backslash\left(C^{\prime} \cap N(0, m)\right)\right)$. We have from (II) that for $t \in C_{*}^{\prime}$ with $|t|>R$

$$
|g(t)| \leq K\left(C^{\prime}, m\right) \exp \left[2 \pi\left(M\left(A y_{t}\right)+\left\langle y_{t}, t\right\rangle\right)\right] .
$$

By Lemma 4 and (31), we have for all $t \in C_{*}^{\prime}$ that

$$
\begin{aligned}
\left\langle y_{t}, t\right\rangle & =\frac{1}{A}\left|M^{-1}\left(\Omega\left(\frac{u_{C}(t)}{A}\right)\right)\right|\left\langle y_{t}^{0}, t\right\rangle \\
& \leq-(1-\eta) \frac{1}{A} u_{C}(t)\left|M^{-1}\left(\Omega\left(\frac{u_{C}(t)}{A}\right)\right)\right| .
\end{aligned}
$$


Since $\left|y_{t}^{0}\right|=1$, we have from (31) that

$$
M\left(A y_{t}\right)=\Omega\left(\frac{u_{C}(t)}{A}\right) .
$$

Applying (35) and (36) to (29), we have for all $t \in C_{*}^{\prime}$ with $|t|>R$ that (37)

$$
|g(t)| \leq K\left(C^{\prime}, m\right) \exp \left[2 \pi \Omega\left(\frac{u_{C}(t)}{A}\right)-2 \pi(1-\eta) \frac{1}{A} u_{C}(t) M^{-1}\left(\Omega\left(\frac{u_{C}(t)}{A}\right)\right)\right] .
$$

Using the Young's inequality in Lemma 6,

$$
\begin{aligned}
u_{C}(t) M^{-1}\left(\Omega\left(\frac{u_{C}(t)}{A}\right)\right) & =A \frac{u_{C}(t)}{A} M^{-1}\left(\Omega\left(\frac{u_{C}(t)}{A}\right)\right) \\
& \leq A\left(M\left(M^{-1}\left(\Omega\left(\frac{u_{C}(t)}{A}\right)\right)\right)+\Omega\left(\frac{u_{C}(t)}{A}\right)\right) \\
& =2 A \Omega\left(\frac{u_{C}(t)}{A}\right) .
\end{aligned}
$$

Applying (38) to (37), we have for all $t \in C_{*}^{\prime}$ with $|t|>R$ that

$$
|g(t)| \leq K\left(C^{\prime}, m\right) \exp \left[-2 \pi(1-2 \eta) \Omega\left(\frac{u_{C}(t)}{A}\right)\right] .
$$

We find the estimation like (39) for $t \in C_{*}^{\prime}$ with $|t| \leq R$ for a fixed $R>0$ of (32). Put

$$
y_{t}^{\prime}=Q y_{t}^{0}
$$

for a $y_{t}^{0} \in \operatorname{Pr}\left(C^{\prime}\right)$ corresponding to $t \in C_{*}^{\prime} \subset C_{*}$ and a fixed $Q>m>0$.

Then since $y_{t}^{0} \in\left(C^{\prime} \backslash\left(C^{\prime} \cap N(0, m)\right)\right)$ and the estimation (29) of (II) holds for $t \in C_{*}^{\prime} \subset C_{*}$ independently of $y \in\left(C^{\prime} \backslash\left(C^{\prime} \cap N(0, m)\right)\right)$, we have from (29), Lemma 4 , and the fact that $\left|y_{t}^{0}\right|=1$ that for $t \in C_{*}^{\prime}$,

(41) $|g(t)| \leq K\left(C^{\prime}, m\right) \exp [2 \pi M(A Q)] \cdot \exp \left[-2 \pi Q(1-\eta) u_{C}(t)\right]$.

Since $\eta \in(0,1)$ and $u_{C}(t)>0$ for $t \in C_{*}^{\prime} \subset C_{*}$, we have that

$$
\exp \left[-2 \pi Q(1-\eta) u_{C}(t)\right] \leq 1, t \in C_{*}^{\prime} \subset C_{*} .
$$

From (41) and Lemma 2, if we take $\eta \in(0,1)$ with $1-2 \eta>0$, we have for $t \in C_{*}^{\prime}$ with $|t| \leq R$ that

(43) $g(t) \leq K\left(C^{\prime}, m\right) \exp [2 \pi M(A Q)] \cdot \exp \left[-2 \pi Q(1-\eta) u_{C}(t)\right]$

$$
\begin{aligned}
\leq & K\left(C^{\prime}, m\right) \exp [2 \pi M(A Q)] \\
= & K\left(C^{\prime}, m\right) \exp [2 \pi M(A Q)] \\
& \cdot \exp \left[2 \pi(1-2 \eta) \Omega\left(\frac{u_{C}(t)}{A}\right)\right] \cdot \exp \left[-2 \pi(1-2 \eta) \Omega\left(\frac{u_{C}(t)}{A}\right)\right] \\
\leq & K\left(C^{\prime}, m\right) \exp [2 \pi M(A Q)]
\end{aligned}
$$




$$
\begin{aligned}
& \cdot \exp \left[2 \pi(1-2 \eta) \Omega\left(\frac{R}{A}\right)\right] \cdot \exp \left[-2 \pi(1-2 \eta) \Omega\left(\frac{u_{C}(t)}{A}\right)\right] \\
= & K\left(C^{\prime}, m\right) C_{A, Q}(\eta) \exp \left[-2 \pi(1-2 \eta) \Omega\left(\frac{u_{C}(t)}{A}\right)\right],
\end{aligned}
$$

where the constant $K\left(C^{\prime}, m\right)$ depends on $C^{\prime}$ and on $m$ and the constant $C_{A, Q}(\eta)$ depends on $\eta$ for two fixed constants $A$ and $Q$. Since $C^{\prime}=C^{\prime}\left(C_{*}^{\prime}, \eta\right)$ depends on $C_{*}^{\prime}$ and on $\eta$ and $m=m\left(C^{\prime}\right)$ depends on $C^{\prime}, K\left(C^{\prime}, m\right) C_{A, Q}(\eta)$ depends on $C_{*}^{\prime}$ and on $\eta$ for two fixed constants $A$ and $Q$. Thus we can find a constant $M\left(C_{*}^{\prime}, \eta\right)$, depending on $C_{*}^{\prime} \subset C_{*}$ and on $\eta$, such that if $t$ is an element of $C_{*}^{\prime} \subset C_{*}$, then $(21)$ holds for any $\eta \in(0,1)$ with $1-2 \eta>0$. This completes the proof of (III).

Proof of (IV). Firstly, in order to show that the Fourier transform in (19) can be taken in the $L_{1}$ sense, we will show that $\left(e^{-2 \pi\langle y, t\rangle} g(t)\right) \in L^{P}, 1 \leq p<\infty$, for $A>0$ and $y \in\left(C^{\prime} \backslash\left(C^{\prime} \cap N(0, m)\right)\right)$. Let $A>0$ and let $p$ be arbitrary with $1 \leq p<\infty$. If we let $y$ be arbitrary but fixed in $\left(C^{\prime} \backslash\left(C^{\prime} \cap N(0, m)\right)\right)$, then $y \in C^{\prime}$ with $|y|>m>0$. Also if we choose a positive real number $\zeta$ such that $0<m /|y|<\zeta<1$, then $\zeta y \in C^{\prime}$ and $|\zeta y|>m$, hence $\zeta y \in\left(C^{\prime} \backslash\left(C^{\prime} \cap N(0, m)\right)\right)$. Then we have from $(29)$ that

$$
\begin{aligned}
\left|e^{-2 \pi\langle y, t\rangle} g(t)\right|^{p} & \leq K\left(C^{\prime}, m\right) e^{-2 \pi p\langle y, t\rangle} \exp [2 \pi p(M(A \zeta y)+\langle\zeta y, t\rangle)] \\
& \leq K\left(C^{\prime}, m\right) \exp [2 \pi p(M(A \zeta y)] \cdot \exp [-2 \pi p((1-\zeta)\langle y, t\rangle)]
\end{aligned}
$$

for all $t \in \mathbb{R}^{n}$. We have from the fact that $(1-\zeta)>0$, Lemma 3 , (40), and [7, p. 39, Theorem 3.2] that

$$
\begin{aligned}
& \int_{\left(C^{*}\right)^{\prime}}\left|e^{-2 \pi\langle y, t\rangle} g(t)\right|^{p} d t \\
\leq & K^{p}\left(C^{\prime}, m\right) \exp [2 \pi p M(A \zeta y)] \int_{\left(C^{*}\right)^{\prime}} \exp [-2 \pi p \delta(1-\zeta)|y||t|] d t \\
\leq & K^{p}\left(C^{\prime}, m\right) Z_{n} \exp [2 \pi p M(A \zeta y)] \int_{0}^{\infty} s^{n-1} \exp [-2 \pi p \delta(1-\zeta)|y| s] d s \\
= & K^{p}\left(C^{\prime}, m\right) Z_{n} \exp [2 \pi p M(A \zeta y)](n-1) !(2 \pi p \delta(1-\zeta)|y|)^{-n} .
\end{aligned}
$$

Here we have used the same techniques as in (18) in the last two steps in (45), where $Z_{n}$ is the area of the unit sphere in $\mathbb{R}^{n}$.

Put $C_{*}^{\prime}=\mathbb{R}^{n} \backslash\left(C^{*}\right)^{\prime}$. Since $C^{*} \subset\left(C^{*}\right)^{\prime}$ and $\left(C^{*}\right)^{\prime}$ is an open cone, $C_{*}^{\prime}$ is a compact subcone of $C_{*}$ and (III) holds for $C_{*}^{\prime}$. Then we have from (14) that

$$
\begin{aligned}
& \int_{C_{*}^{\prime}}\left|e^{-2 \pi\langle y, t\rangle} g(t)\right|^{p} d t \\
\leq & K^{\prime}\left(C_{*}^{\prime}, A, \eta\right) \exp \left[2 \pi p T \rho_{C} \frac{A}{\xi} \cdot \omega^{-1}\left(\frac{T \rho_{C}}{1-3 \eta}\right)\right. \\
& \left.-2 \pi p(1-\eta) \Omega\left(\omega^{-1}\left(\frac{T \rho_{C}}{1-3 \eta}\right)\right)\right],
\end{aligned}
$$


where $K^{\prime}\left(C_{*}^{\prime}, A, \eta\right)$ is a constant depending on $C_{*}^{\prime}$, on a fixed $\eta \in(0,1)$, and on a fixed $A>0$. Here $\xi=\xi\left(C_{*}^{\prime}\right)$ is the number in Lemma 2 .

The open cone $\left(C^{*}\right)^{\prime}$ in (45) is fixed depending on the compact subcone $C^{\prime} \subset \mathcal{O}(C)$. Then the compact subcone $C_{*}^{\prime} \subset C_{*}$ in (46) was defined by $C_{*}^{\prime}=\mathbb{R}^{n} \backslash\left(C^{*}\right)^{\prime}$. Since $\left(C^{*}\right)^{\prime} \cup C_{*}^{\prime}=\mathbb{R}^{n}$ and $\left(C^{*}\right)^{\prime} \cap C_{*}^{\prime}=\emptyset$, we have from (45) and (46) that $\left(e^{-2 \pi\langle y, t\rangle} g(t)\right) \in L^{P}, 1 \leq p<\infty$, for $A>0$ and $y \in$ $\left(C^{\prime} \backslash\left(C^{\prime} \cap N(0, m)\right)\right)$.

Now if $A=0$, then $g(t)$ satisfies (29) and supp $(g) \subseteq C^{*}$. The open cone $\left(C^{*}\right)^{\prime}$ for which Lemma 3 holds contains $C^{*}$, hence we have from (45) that $\left(e^{-2 \pi\langle y, t\rangle} g(t)\right) \in L^{P}, 1 \leq p<\infty$, for $y \in\left(C^{\prime} \backslash\left(C^{\prime} \cap N(0, m)\right)\right)$.

Thus for either of the cases $A>0$ or $A=0$, the Fourier transform in (15) can be taken in the $L_{1}$ sense.

Secondly, we show that $\left\langle V, e^{2 \pi\langle z, t\rangle}\right\rangle$ is well defined on $T\left(C^{\prime} ; m\right)$. We consider

$$
\int_{\mathbb{R}^{n}} g(t) e^{2 \pi i\langle z, t\rangle} d t, \quad z \in T\left(C^{\prime} ; m\right) .
$$

Since $\left(C^{*}\right)^{\prime} \cap C_{*}^{\prime}=\emptyset$ and $\left(C^{*}\right)^{\prime} \cup C_{*}^{\prime}=\mathbb{R}^{n},(47)$ can be rewrite as

$$
\begin{aligned}
\int_{\mathbb{R}^{n}} g(t) e^{2 \pi i\langle z, t\rangle} d t & =\int_{C_{*}^{\prime}} g(t) e^{2 \pi i\langle z, t\rangle} d t+\int_{\left(C^{*}\right)^{\prime}} g(t) e^{2 \pi i\langle z, t\rangle} d t \\
& =h_{C_{*}^{\prime}}^{0, g}+h_{\left(C^{*}\right)^{\prime}}^{0, g}
\end{aligned}
$$

Here $h_{C_{*}^{\prime}}^{0, g}$ and $h_{\left(C^{*}\right)}^{0, g}$, are the functions corresponding to $(\gamma, g)=(0, g)$ in Lemma 12 and Lemma 13 , respectively. Since $T\left(C^{\prime} ; m\right) \subset T^{C^{\prime}}, h_{C_{*}^{\prime}}^{0, g}$ converges absolutely and uniformly on $T^{C^{\prime}}$ by Lemma 12 and $h_{\left(C^{*}\right)}^{0, g}$, converges absolutely and uniformly on $T\left(C^{\prime} ; m\right)$ by Lemma $13,\left\langle V, e^{2 \pi\langle z, t\rangle}\right\rangle$ is well-defined on $T\left(C^{\prime} ; m\right)$.

Hence since the Fourier transform in (19) can be taken in the $L_{1}$ sense and $\left\langle V, e^{2 \pi i\langle z, t\rangle}\right\rangle$ is well-defined on $T\left(C^{\prime} ; m\right)$, if we use differentiation in the distributional sense, then we have that

$$
\begin{aligned}
\left\langle V, e^{2 \pi i\langle z, t\rangle}\right\rangle & =(-1)^{|\alpha|}\left\langle g(t), D_{t}^{\alpha}\left(e^{2 \pi i\langle z, t\rangle}\right)\right\rangle \\
& =z^{\alpha} \int_{\mathbb{R}^{n}} g(t) e^{2 \pi i\langle z, t\rangle} d t=z^{\alpha} \mathcal{F}\left[e^{-2 \pi\langle y, t\rangle} g(t) ; x\right]
\end{aligned}
$$

for $z \in T\left(C^{\prime} ; m\right)$ and the Fourier transform is taken in either the $L^{1}$ or $L^{2}$ sense. From (19) and (49) we have (22). This completes the proof of (IV).

Proof of $(\mathrm{V})$. The proof of $(\mathrm{V})$ follows from only replacing $\mathcal{K}_{r}, \mathbf{K}_{\mathbf{r}}, \mathcal{K}_{r}^{\prime}$, and $\mathbf{K}_{\mathbf{r}}^{\prime}$ in proving (7.3) of [3, pp. 1056-1057] by $\mathcal{K}_{M}, \mathbf{K}_{\mathbf{M}}, \mathcal{K}_{M}^{\prime}$, and $\mathbf{K}_{\mathbf{M}}^{\prime}$, respectively.

Proof of (VI). Firstly, we show that $\left\{e^{-2 \pi\langle y, t\rangle} V_{t}: y \in\left(C^{\prime} \backslash\left(C^{\prime} \cap N(0, m)\right)\right)\right.$, $\left.|y| \leq Q_{m}\right\}, Q_{m}>M>0$, is strongly bounded set in $\mathcal{K}_{M}^{\prime}$. Let $\Phi$ be an arbitrary bounded set in $\mathcal{K}_{M}$ and let $\phi \in \Phi$. Since $\left(e^{-2 \pi\langle y, t\rangle} V_{t}\right) \in \mathcal{K}_{M}^{\prime}$ for any $y \in \mathbb{R}^{n}$, we have from Lemma 8 and general Leibnitz rule that for some $n$-tuple 
$\alpha$ of nonnegative integers, some integer $k \geq 0$, and some continuous function $f$ on $\mathbb{R}^{n}$ bounded by $M>0$,

$$
\begin{aligned}
& \left\langle e^{-2 \pi\langle y, t\rangle} V_{t}, \phi(t)\right\rangle \\
= & \left\langle D_{t}^{\alpha}(\exp [M(k t) f(t)]), e^{-2 \pi\langle y, t\rangle} \phi(t)\right\rangle \\
= & (-1)^{|\alpha|} \int_{\mathbb{R}^{n}} e^{M(k t)} f(t) \sum_{\beta+\gamma=\alpha} \frac{\alpha !}{\beta ! \gamma !}\left(\frac{1}{i}\right)^{|\beta|} y^{\beta} e^{-2 \pi\langle y, t\rangle} D_{t}^{\gamma}(\phi(t)) d t \\
= & (-1)^{|\alpha|} \sum_{\beta+\gamma=\alpha} \frac{\alpha !}{\beta ! \gamma !}\left(\frac{1}{i}\right)^{|\beta|} y^{\beta} I_{y}(\gamma),
\end{aligned}
$$

where

$$
I_{y}(\gamma)=\int_{\mathbb{R}^{n}} e^{M(k t)} f(t) e^{-2 \pi\langle y, t\rangle} D_{t}^{\gamma}(\phi(t)) d t .
$$

Let $Q_{m}>0$ be an arbitrary but fixed real number. For $y \in \mathbb{R}^{n}$ with $|y| \leq Q_{m}$, if we choose $r \geq \max \left\{|\alpha|, 2 k+2 \pi Q_{m}\right\}$, we have from Lemma 5 and the fact that $\phi \in \mathcal{K}_{M}$ that

$$
\begin{aligned}
\left|I_{y}(\gamma)\right| & \leq M \int_{\mathbb{R}^{n}} e^{M(k t)} e^{2 \pi|y||t|}\left|D_{t}^{\gamma}(\phi(t))\right| d t \\
& \leq M \int_{\mathbb{R}^{n}} e^{M(k t)} e^{2 \pi Q_{m}|t|}\left|D_{t}^{\gamma}(\phi(t))\right| d t \\
& \leq M \int_{\mathbb{R}^{n}} e^{-M(k t)} e^{M(2 k t)} e^{M\left(2 \pi Q_{m} t\right)}\left|D_{t}^{\gamma}(\phi(t))\right| d t \\
& \leq M \int_{\mathbb{R}^{n}} e^{M\left(\left(2 k+2 \pi Q_{m}\right) t\right)}\left|D_{t}^{\gamma}(\phi(t))\right| e^{-M(k t)} d t \\
& \leq M\|\phi\|_{\mathcal{K}_{M}} \int_{\mathbb{R}^{n}} e^{-M(k t)} d t,
\end{aligned}
$$

where $M$ is such that $\sup _{t \in \mathbb{R}^{n}}|f(t)| \leq M$. Since $\Phi$ is a bounded set in $\mathcal{K}_{M}$, there exist a constant $W_{\gamma}$, depending only $\gamma$, such that $\|\phi\|_{\mathcal{K}_{M}} \leq W_{\gamma}$ for all $\phi \in \Phi$. Hence for each $\gamma$ with $\beta+\gamma=\alpha$,

$$
\left|I_{y}(\gamma)\right| \leq M W_{\gamma} \int_{\mathbb{R}^{n}} e^{-M(k t)} d t=W_{\gamma}^{\prime}
$$

for all $\phi \in \Phi$. Thus we have from (50) and (53) that

$$
\left|\left\langle e^{-2 \pi\langle y, t\rangle} V_{t}, \phi(t)\right\rangle\right| \leq W_{\gamma}^{\prime} \sum_{\beta+\gamma=\alpha} \frac{\alpha !}{\beta ! \gamma !} Q_{m}^{|\beta|}, \quad \phi \in \Phi .
$$

Here the bound in (54) is independent of $\phi \in \Phi$. Hence $\left\{e^{-2 \pi\langle y, t\rangle} V_{t}: y \in\right.$ $\left.\left(C^{\prime} \backslash\left(C^{\prime} \cap N(0, m)\right)\right),|y| \leq Q_{m}\right\}, Q_{m}>M>0$, is a bounded set in complex plane. Since $\Phi$ be an arbitrary bounded set in $\mathcal{K}_{M},\left\{e^{-2 \pi\langle y, t\rangle} V_{t}: y \in\left(C^{\prime} \backslash\left(C^{\prime} \cap\right.\right.\right.$ 
$\left.N(0, m))),|y| \leq Q_{m}\right\}, Q_{m}>M>0$, is strongly bounded set in $\mathcal{K}_{M}^{\prime}$. Thus we have from Lemma 10 and (23) that

$$
\begin{aligned}
& \left\{f(z): y=\operatorname{Im}(z) \in\left(C^{\prime} \backslash\left(C^{\prime} \cap N(0, m)\right)\right),|y| \leq Q_{m}\right\} \\
= & \left\{\mathcal{F}\left[e^{-2 \pi\langle y, t\rangle} V_{t}\right]: y=\operatorname{Im}(z) \in\left(C^{\prime} \backslash\left(C^{\prime} \cap N(0, m)\right)\right),|y| \leq Q_{m}\right\}
\end{aligned}
$$

is strongly bounded set in $\mathbf{K}_{\mathbf{M}}^{\prime}$. This completes the proof of (VI).

We consider the converse of Theorem 1 . We note that the inequality (20) in Theorem 1 can be rewrite as

$$
|g(t)| \leq K_{g} e^{k_{g}|t|}, \quad t \in \mathbb{R}^{n},
$$

for some two positive constants $K_{g}$ and $k_{g}$ both of which are depend on $g$. We will use the inequality (55) instead of the inequality (20) in the next theorem.

Theorem 2. Let $C$ be an open connected cone in $\mathbb{R}^{n}$ and let $C_{*}^{\prime}$ be an arbitrary compact subcone of $C_{*}=\mathbb{R}^{n} \backslash C^{*}$. Let $A>0$ be such that $A / \xi \leq 1$, where $\xi=\xi\left(C_{*}^{\prime}\right)$ is a constant, depending on $C_{*}^{\prime}$, as in Lemma 2. Let $V$ be a finite sum

$$
V=\sum_{\alpha} D_{t}^{\alpha}\left(g_{\alpha}(t)\right)
$$

where each $g_{\alpha}$ are continuous function of $t \in \mathbb{R}^{n}$. Assume that for each $n$-tuple of nonnegative integers $\alpha, g_{\alpha}(t)$ satisfies

$$
\left|g_{\alpha}(t)\right| \leq K_{\alpha} e^{k_{\alpha}|t|}, \quad t \in \mathbb{R}^{n},
$$

where some two positive constants $K_{\alpha}$ and $k_{\alpha}$ both of which are depend on $g_{\alpha}$. Also assume that each $g_{\alpha}$ satisfies

$$
\left|g_{\alpha}(t)\right| \leq M\left(C_{*}^{\prime}, \eta\right) \exp \left[-2 \pi(1-2 \eta) \Omega\left(\frac{u_{C}(t)}{A}\right)\right], t \in C_{*}^{\prime} \subset C_{*},
$$

for any $\eta \in(0,1)$ with $1-3 \eta>0$, where $M\left(C_{*}^{\prime}, \eta\right)$ is a constant depending on $C_{*}^{\prime}$ and on $\eta$. Then $V \in \mathcal{K}_{1}^{\prime} \subset \mathcal{K}_{M}^{\prime}$. Furthermore the function

$$
f(z)=\left\langle V, e^{2 \pi i\langle z, t\rangle}\right\rangle
$$

and any derivative of $f(z)$ belong to $G_{M}\left(\frac{\rho_{C}}{1-3 \eta} ; \mathcal{O}(C)\right)$.

Proof. Since $g_{\alpha}(t)$ is continuous and $g_{\alpha}(t) \in \mathcal{K}_{1}^{\prime} \subset \mathcal{K}_{M}^{\prime}, V \in \mathcal{K}_{1}^{\prime} \subset \mathcal{K}_{M}^{\prime}$. Using the differentiation in the distribution sense, we write $f(z)$ as

$$
f(z)=\left\langle V, e^{2 \pi i\langle z, t\rangle}\right\rangle=\sum_{\alpha} z^{\alpha} \int_{\mathbb{R}^{n}} g_{\alpha}(t) e^{2 \pi i\langle z, t\rangle} d t .
$$

To show the existence and analyticity of $f(z)$ for a certain $z$, consider

$$
h_{\alpha}(z)=\int_{\mathbb{R}^{n}} g_{\alpha}(t) e^{2 \pi i\langle z, t\rangle} d t .
$$


Since $\left(C^{*}\right)^{\prime} \cap C_{*}^{\prime}=\emptyset$ and $\left(C^{*}\right)^{\prime} \cup C_{*}^{\prime}=\mathbb{R}^{n},(60)$ can be rewritten by

$$
\begin{aligned}
h_{\alpha}(z) & =\int_{\mathbb{R}^{n}} g_{\alpha}(t) e^{2 \pi i\langle z, t\rangle} d t \\
& =\int_{C_{*}^{\prime}} g_{\alpha}(t) e^{2 \pi i\langle z, t\rangle} d t+\int_{\left(C^{*}\right)^{\prime}} g_{\alpha}(t) e^{2 \pi i\langle z, t\rangle} d t \\
& =h_{C_{*}^{\prime}}^{0, \alpha}(z)+h_{\left(C^{*}\right)^{\prime}}^{0, \alpha}(z),
\end{aligned}
$$

where $h_{C_{*}^{\prime}}^{0, \alpha}(z)$ and $h_{\left(C^{*}\right)^{\prime}}^{0, \alpha}(z)$ are the functions corresponding to $(\gamma, g)=\left(0, g_{\alpha}\right)$ in Lemma 12 and Lemma 13, respectively.

Also we have from (59), the generalized Leibnitz rule, and the fact that $T\left(C^{\prime} ; m\right) \subset T^{C^{\prime}}$ that

$$
\begin{aligned}
D_{z}^{\gamma}(f(z)) & =\sum_{\alpha} \sum_{\beta+\mu=\gamma} \frac{\gamma !}{\beta ! \mu !} D_{z}^{\beta}\left(z^{\alpha}\right)\left[D_{z}^{\mu}\left(\left(h_{C_{*}^{\prime}}^{0, \alpha}\right)(z)\right)+D_{z}^{\mu}\left(\left(h_{\left(C^{*}\right)^{\prime}}^{0, \alpha}\right)(z)\right)\right] \\
& =\sum_{\alpha} \sum_{\beta+\mu=\gamma} \frac{\gamma !}{\beta ! \mu !} D_{z}^{\beta}\left(z^{\alpha}\right)(-1)^{|\mu|}\left[h_{C_{*}^{\prime}}^{\gamma, \alpha}(z)+h_{\left(C^{*}\right)^{\prime}}^{\gamma, \alpha}(z)\right], z \in T\left(C^{\prime} ; m\right),
\end{aligned}
$$

where $\gamma, \beta$ and $\mu$ are $n$-tuples of nonnegative integers. Here $h_{C^{\prime}}^{\gamma, \alpha}(z)$ and $h_{\left(C^{*}\right)^{\prime}}^{\gamma, \alpha}(z)$ are the functions corresponding to $(\gamma, g)=\left(\gamma, g_{\alpha}\right)$ in Lemma 12 and Lemma 13, respectively.

Let $C^{\prime}$ be an arbitrary compact subcone of $\mathcal{O}(C)$. Choose $m_{\alpha}=m_{\alpha}\left(C^{\prime}\right)$, depending on $\alpha$ and on $C^{\prime}$, such that

$$
m_{\alpha}=\left(k_{\alpha} /(2 \pi \delta)\right)+1
$$

where $k_{\alpha}$ is as in (57) and $\delta$ is as in Lemma 3. For $m_{\alpha}>0$ in (63), let $z_{0}$ be an arbitrary but fixed point in $T\left(C^{\prime} ; m_{\alpha}\right)=\mathbb{R}^{n}+i\left(C^{\prime} \backslash\left(C^{\prime} \cap N\left(0, m_{\alpha}\right)\right)\right)$. If we choose an open neighborhood $N^{\prime}\left(z_{0}, r\right)$ of $z_{0}$ with radius $r>0$ whose closure is contained in $T\left(C^{\prime} ; m_{\alpha}\right) \subset T^{C^{\prime}}, h_{C_{*}^{\prime}}^{\gamma, \alpha}(z)$ and $h_{\left(C^{*}\right)^{\prime}}^{\gamma, \alpha}(z)$ converge absolutely and uniformly for $z \in N^{\prime}\left(z_{0}, r\right)$ from Lemma 12 and Lemma 13, respectively. Since $z$ is an arbitrary point in $T\left(C^{\prime} ; m_{\alpha}\right)$, we have from $(62)$ that $f(z)$ and its derivative is analytic in $T\left(C^{\prime} ; m_{\alpha}\right)$.

We put

$$
m=\max _{\alpha}\left\{m_{\alpha}\right\}
$$

where $m_{\alpha}$ is as in (63) for each $\alpha$. Since $T\left(C^{\prime} ; m\right) \subset T\left(C^{\prime} ; m_{\alpha}\right), h_{\alpha}(z)$ in (61) is analytic in $T\left(C^{\prime} ; m\right)$ for each $\alpha$, hence $\sum_{\alpha} z^{\alpha} h_{\alpha}(z)$ is also analytic in $T\left(C^{\prime} ; m\right)$. Thus $f(z)=\left\langle V, e^{2 \pi i\langle z, t\rangle}\right\rangle$ and any derivative of $f(z)$ are analytic in $T\left(C^{\prime} ; m\right), C^{\prime} \subset \mathcal{O}(C)$, for the fixed $m$ in (64).

Now we will obtain a growth of $f(z)$ and any derivative of $f(z)$ like the inequality in the definition of $G_{M}(A ; C)$ for any compact subcone $C^{\prime} \subset \mathcal{O}(C)$ and the corresponding $m>0$ taken in (64). 
In order to estimate integral representations of $f(z)$ and any derivative of $f(z)$ of the form (59) on $C_{*}^{\prime}$, we will continue the estimation of inequalities in (14) under the additional condition of $A$ and $\xi$ in this theorem.

Let $A>0$ be such that $A / \xi \leq 1$, where $\xi=\xi\left(C_{*}^{\prime}\right)$ is a constant, depending on $C_{*}^{\prime}$, as in Lemma 2 and let $z \in N^{\prime}\left(z_{0}, r\right) \subset T^{C^{\prime}}$, where $N^{\prime}\left(z_{0}, r\right)$ is an open neighborhood of $z_{0}$ with radius $r>0$ whose closure is in $T^{C^{\prime}}$.

If we replace $T$ by $|y|$ in (11), (12), and (13), we have from (14) that

$$
\begin{aligned}
\left|h_{C_{*}^{\prime}}^{\gamma, \alpha}(z)\right|= & \left|\int_{C_{*}^{\prime}} t^{\gamma} g(t) e^{2 \pi i\langle z, t\rangle} d t\right| \\
\leq & K^{\prime}\left(C_{*}^{\prime}, A, \eta\right) \exp \left[2 \pi|y| \rho_{C} \frac{A}{\xi} \cdot \omega^{-1}\left(\frac{|y| \rho_{C}}{1-3 \eta}\right)\right. \\
& \left.-2 \pi(1-3 \eta) \Omega\left(\omega^{-1}\left(\frac{|y| \rho_{C}}{1-3 \eta}\right)\right)\right]
\end{aligned}
$$

for $n$ tuples $\gamma$ and $\alpha$ of nonnegative integers and all $z \in N^{\prime}\left(z_{0}, r\right)$, where $K^{\prime}\left(C_{*}^{\prime}, A, \eta\right)$ is a constant depending on fixed $C_{*}^{\prime}$, on fixed $A>0$, and on fixed $\eta \in(0,1)$ with $1-3 \eta>0$. By Lemma 6 ,

$$
\frac{|y| \rho_{C}}{1-3 \eta} \cdot \omega^{-1}\left(\frac{|y| \rho_{C}}{1-3 \eta}\right)=M\left(\frac{|y| \rho_{C}}{1-3 \eta}\right)+\Omega\left(\omega^{-1}\left(\frac{|y| \rho_{C}}{1-3 \eta}\right)\right) .
$$

Since $A / \xi \leq 1$ and $0<1-3 \eta<1$, we have from (66) that

$$
\begin{aligned}
& |y| \rho_{C} \frac{A}{\xi} \cdot \omega^{-1}\left(\frac{|y| \rho_{C}}{1-3 \eta}\right)-(1-3 \eta) \Omega\left(\omega^{-1}\left(\frac{|y| \rho_{C}}{1-3 \eta}\right)\right) \\
\leq & |y| \rho_{C} \cdot \omega^{-1}\left(\frac{|y| \rho_{C}}{1-3 \eta}\right)-(1-3 \eta) \Omega\left(\omega^{-1}\left(\frac{|y| \rho_{C}}{1-3 \eta}\right)\right) \\
= & (1-3 \eta) M\left(\frac{|y| \rho_{C}}{1-3 \eta}\right) \leq M\left(\frac{|y| \rho_{C}}{1-3 \eta}\right) .
\end{aligned}
$$

Applying (67) to (65), since $z$ is an arbitrary point in $T^{C^{\prime}}$, we have that for $n$ tuples $\gamma$ and $\alpha$ of nonnegative integers

$$
\left|h_{C_{*}^{\prime}}^{\gamma, \alpha}(z)\right| \leq K^{\prime}\left(C_{*}^{\prime}, A, \eta\right) \exp \left[M\left(\frac{|y| \rho_{C}}{1-3 \eta}\right)\right], \quad z \in T^{C^{\prime}},
$$

where $K^{\prime}\left(C_{*}^{\prime}, A, \eta\right)$ is a constant depending on fixed $C_{*}^{\prime}$, on fixed $A>0$ with $A / \xi \leq 1$, and on fixed $\eta \in(0,1)$ with $1-3 \eta>0$.

We now consider the integral representations of $f(z)$ and any derivative of $f(z)$ in (59) on $\left(C^{*}\right)^{\prime}$. Let $m_{\alpha}$ and $m$ be as in (63) and (64), respectively. Since $m \geq m_{\alpha}$ for each $m_{\alpha}, k_{\alpha}-2 \pi \delta|y|<-2 \pi \delta<0$ when $|y|>m$ and $y \in C^{\prime}$. Then we have from Lemma 13 that for $n$ tuples $\gamma$ and $\alpha$ of nonnegative integers,

$$
\left|h_{\left(C^{*}\right)^{\prime}}^{\gamma, \alpha}(z)\right|=\left|\int_{\left(C^{*}\right)^{\prime}}\right| t^{\gamma}\left|g(t) e^{2 \pi i\langle z, t\rangle} d t\right|
$$




$$
\begin{aligned}
& \leq K_{\alpha} Z_{n}(|\gamma|+n-1) !(2 \pi \delta)^{-|\gamma|-n} \\
& =Q_{\alpha}\left(m, C^{\prime}\right)<\infty, \quad z \in T\left(C^{\prime} ; m\right),
\end{aligned}
$$

where $Q_{\alpha}\left(m, C^{\prime}\right)$ is a constant depending on $m$ chosen in (64) and on $C^{\prime} \subset$ $\mathcal{O}(C)$ since $\delta=\delta\left(C^{\prime}\right)$ depends on $C^{\prime}$.

Applying (68) and (69) to (62), we can find a nonnegative real number $N$ which does not depend on $m$ chosen in (64) or on $C^{\prime}$ such that for $n$ tuples $\gamma$ and $\alpha$ of nonnegative integers and $z \in T\left(C^{\prime} ; m\right)$,

$$
\begin{aligned}
\left|D_{z}^{\gamma}(f(z))\right| \leq & \sum_{\alpha} C_{\alpha}(1+|z|)^{N} \\
& \cdot\left[K^{\prime}\left(C_{*}^{\prime}, A, \eta\right) \exp \left[M\left(\frac{|y| \rho_{C}}{1-3 \eta}\right)\right]+Q_{\alpha}\left(m, C^{\prime}\right)\right] \\
\leq & K_{1}\left(m, C^{\prime}, A, \eta\right)(1+|z|)^{N} \exp \left[M\left(\frac{|y| \rho_{C}}{1-3 \eta}\right)\right]
\end{aligned}
$$

where $K_{1}\left(m, C^{\prime}, A, \eta\right)$ is a constant depending on $m$ chosen in (64), on $C^{\prime}$, on fixed $A>0$ with $A / \xi \leq 1$, and on fixed $\eta \in(0,1)$ with $1-3 \eta>0$. Here we note that $C_{*}^{\prime}=\mathbb{R}^{n} \backslash\left(C^{*}\right)^{\prime}$ depends on $C^{\prime} \subset \mathcal{O}(C)$. This complete the proof of Theorem 2.

We can extend the results that are described from last paragraph of $[3$, p. 1060] to Corollary 7.1 of [3, p. 1061] to the results in the context of spaces $\mathcal{K}_{M}^{\prime}$ or spaces $G_{M}(A ; C)$ by the exactly same line there as follow;

(i) Under the hypothesis of Theorem 2, (V) and (VI) are hold for $z \in$ $T\left(C^{\prime} ; m\right), C^{\prime} \subset \mathcal{O}, m=m\left(C^{\prime}\right)>0$.

(ii) Let $C$ be an open connected cone and let $C_{*}^{\prime}$ be an arbitrary compact subcone of $C_{*}=\mathbb{R}^{n} \backslash C^{*}$. Let $A>0$ be such that $A / \xi \leq 1$, where $\xi=\xi\left(C_{*}^{\prime}\right)$ is a constant, depending on $C_{*}^{\prime}$, as in Lemma 2. If $f(z) \in G_{M}(A ; C)$, then $f(z)$ and any derivative of $f(z)$ can be extended to an element of $G_{M}\left(\frac{\rho_{C}}{1-3 \eta} ; \mathcal{O}(C)\right)$ for a constant $\eta \in(0,1)$ with $1-3 \eta>0$.

\section{The relationship between $F_{M}(A ; C), \mathcal{K}_{M}^{\prime}$, and $K_{M}^{\prime}$ and distributional boundary values of the spaces $F_{M}(A ; C)$}

In this section, we only state without proof the relationship between $F_{M}(A ; C), \mathcal{K}_{M}^{\prime}$, and $\mathbf{K}_{\mathbf{M}}^{\prime}$ since the ideas, methods, and any others needed to obtain the relationship between $F_{M}(A ; C), \mathcal{K}_{M}^{\prime}$, and $\mathbf{K}_{\mathbf{M}}^{\prime}$ are the same as that of obtaining the relationship between $G_{M}(A ; C), \mathcal{K}_{M}^{\prime}$ and $\mathbf{K}_{\mathbf{M}}^{\prime}$ in the previous section.

Exceptionally, we show that the elements of the spaces $F_{M}(A ; C)$ can obtain distributional boundary values in $\mathbf{K}_{\mathbf{M}}^{\prime}$.

Theorem 3. Let $M(x)$ and $\Omega(y)$ be the functions as in Definition 7 . For the open connected cone $C$, let $f(z) \in F_{M}(A ; C)$. For any compact subcone $C^{\prime} \subset C$, let $m=m\left(C^{\prime}\right)$ be a fixed real number which depends on $C^{\prime}$ as in the 
definition of $F_{M}(A ; C)$. Then there exist a unique element $V=D_{t}^{\alpha}(g(t)) \in$ $\mathcal{K}_{M}^{\prime}$, where $\alpha$ is an $n$-tuple of nonnegative integers and $g(t)$ is a continuous function of $t \in \mathbb{R}^{n}$ such that the following are hold.

(I) For $A \geq 0$

$$
f(z)=z^{\alpha} \mathcal{F}\left[e^{-2 \pi\langle y, t\rangle} g(t) ; x\right], \quad z=x+i y \in T^{C^{\prime}},
$$

where the Fourier transform is taken in the $L^{2}$ sense.

(II) For $A \geq 0, g(t)$ satisfies

$$
|g(t)| \leq K\left(C^{\prime}, m\right) \exp [2 \pi(M(A y)+|y||t|)], \quad t \in \mathbb{R}^{n},
$$

where $C^{\prime} \subset C$ is arbitrary and $K\left(C^{\prime}, m\right)$ depends on $C^{\prime}$ and on $m$. Inequality (71) is independent of $y \in\left(C^{\prime} \backslash\left(C^{\prime} \cap N(0, m)\right)\right)$ and $\operatorname{supp}(g)=\operatorname{supp}(V) \subseteq\{t$ : $\left.u_{C}(t) \leq A\right\}$.

(III) For $A>0$ and any compact subcone of $C_{*}^{\prime} \subset C_{*}=\mathbb{R}^{n} \backslash C^{*}, g(t)$ satisfies

$$
|g(t)| \leq M\left(C_{*}^{\prime}, \eta\right) \exp \left[-2 \pi(1-2 \eta) \Omega\left(\frac{u_{C}(t)}{A}\right)\right], t \in C_{*}^{\prime},
$$

for any $\eta \in(0,1)$ with $1-2 \eta>0$, where $M\left(C_{*}^{\prime}, \eta\right)$ is a constant depending on $C_{*}^{\prime}$ and on $\eta$.

(IV) For $A \geq 0$, if $g(t)$ satisfies that $|g(t)| \leq K e^{k|t|}, t \in\left(C^{*}\right)^{\prime}$, for some constant $K$ and $k>0$, then

$$
f(z)=\left\langle V, e^{2 \pi i\langle z, t\rangle}\right\rangle, \quad z=x+i y \in T^{C^{\prime}} .
$$

(V) For $A \geq 0$,

$$
f(z)=\mathcal{F}\left[e^{-2 \pi\langle y, t\rangle} V_{t}\right], \quad z=x+i y \in T^{C^{\prime}},
$$

where the equality in (72) holds in $\mathbf{K}_{\mathbf{M}}^{\prime}$.

(VI)

$$
\left\{f(z): y=\operatorname{Im}(z) \in\left(C^{\prime} \backslash\left(C^{\prime} \cap N(0, m)\right)\right),|y| \leq Q_{m}\right\}
$$

is strongly bounded in $\mathbf{K}_{\mathbf{M}}^{\prime}$, where $Q_{m}>m>0$.

(VII) $f(z) \rightarrow \mathcal{F}[V] \in \mathbf{K}_{\mathbf{M}}^{\prime}$ in the strong and weak topology of $\mathbf{K}_{\mathbf{M}}^{\prime}$ as $y=$ $\operatorname{Im}(z) \rightarrow 0, y \in C^{\prime} \subset C$, where this boundary value is obtained independently of how $y \rightarrow 0$ in $C^{\prime} \subset C$.

Proof. It suffices to prove only (VII). Since $V \in \mathcal{K}_{M}^{\prime}$, if we replace $\mathcal{K}_{p}^{\prime}$ and $e^{k|t|^{p}}$ in the proof of Lemma 5.9 in [3, pp. 1052-1053] by $\mathcal{K}_{M}^{\prime}$ and $e^{M(k t)}$, respectively, we have that

$$
\lim _{y \rightarrow 0} e^{-2 \pi\langle y, t\rangle} V_{t}=V_{t}, \quad y \in \mathbb{R}^{n},
$$

in the weak topology of $\mathcal{K}_{M}^{\prime}$. Since $\mathcal{K}_{M}$ is a Montel space by Lemma 7 , we also have the convergence (73) in the strong topology of $\mathcal{K}_{M}^{\prime}$. Since the Fourier transform is a topological isomorphism of $\mathcal{K}_{M}^{\prime}$ onto $\mathbf{K}_{\mathbf{M}}^{\prime}$ by Lemma $10, f(z) \rightarrow$ $\mathcal{F}[V] \in \mathbf{K}_{\mathbf{M}}^{\prime}$ in the strong and weak topology of $\mathbf{K}_{\mathbf{M}}^{\prime}$ as $y=\operatorname{Im}(z) \rightarrow 0, y \in$ $C^{\prime} \subset C$. Since $V$ is independent of how $y \rightarrow 0$ in $C^{\prime} \subset C$, the boundary value 
$\mathcal{F}[V]$ is obtained independently of how $y \rightarrow 0$ in $C^{\prime} \subset C$. This completes the proof of (VII).

Acknowledgement. The author would like to thank the referees for their careful reading and valuable comments given to improve the paper.

\section{References}

[1] R. D. Carmichael, Distributional boundary values of functions analytic in tubular radial domains, Indiana Univ. Math. J. 20 (1971), 843-853.

[2] $\longrightarrow$ Distributions of exponential growth and their Fourier transforms, Duke Math. J. 40 (1973), 765-783.

[3] _ Analytic functions related to the distributions of exponential growth, SIAM J. Math. Anal. 10 (1979), no. 5, 1041-1068.

[4] I. M. Gel'fand and G. E. Shilov, Generalized Functions, vol. 3, Academic Press, New York, 1968

[5] D. H. Pahk Structure theorem and Fourier transform for distributions with restricted growth, Kyungpook Math. J. 23 (1983), no. 2, 129-146.

[6] G. Sampson and Z. Zielezny, Hypoellitic convolution equations in $\mathcal{K}_{p}^{\prime}, p>1$, Trans. Amer. Math. Soc. 223 (1976), 133-154.

[7] L. Schwarz, Mathematics for the Physical Sciences, Addison-Wesley, Reading, MA, 1966.

[8] J. Sevastião and E. Silva, Le calcul opérationnel au point de vue desdistributions, Portugal. Math. 14 (1955), 105-132.

[9] V. S. Vladimirov, Methods of the Theory of Functions of Many Complex Variables, M. I. T. Press, Cambridge, MA, 1966.

Department of Mathematics

INJE UNIVERSITY

Kimhae 621-749, Korea

E-mail address: mathsohn@inje.ac.kr 